\title{
Superior Vena Cava Syndrome
}

\author{
Brian Funaki, M.D. ${ }^{1}$
}

Superior vena cava syndrome occurs when there is acute or subacute stenosis or occlusion of the superior vena cava. Marked facial and upper extremity swelling results in dysphagia, dyspnea, and pain. Both benign and malignant processes can cause superior vena cava syndrome; the most common etiologies are tumor, indwelling catheters, and mediastinal fibrosis. The acuity of the obstructive process has important ramifications: a slowly occlusive process may allow collateral venous drainage (as seen in some hemodialysis patients) and remain relatively well compensated, and a rapid occlusion nearly always results in severe symptoms.

\section{CASE REPORT}

A 68-year-old man with acute lymphocytic leukemia was referred to the Vascular and Interventional Radiology Section for dual lumen subcutaneous chest port insertion for chemotherapy (Fig. 1). This procedure was uneventful and chemotherapy was initiated soon after port insertion. After 6 weeks, the patient experienced rapidonset swelling of both upper extremities and face. He underwent an enhanced computed tomography (CT) examination for suspected superior vena cava syndrome, which revealed narrowing and partial thrombosis of the superior vena cava at the site of the catheter tip with retrograde flow into the azygous vein (Fig. 2).

The patient underwent a short course of thrombolytic therapy with no improvement in symptoms. After consultation with the Interventional Radiology service, port removal and superior vena cava angioplasty were planned. Informed consent was obtained. Because the patient's symptoms were exacerbated in the supine position, sedation was administered and supervised by an anesthesiologist. The right chest was prepped and draped in usual fashion (Fig. 3A). Blunt and sharp dissections were performed to free the port from the subcutaneous tissue of the chest. The catheter portion was detached from the hub and a 0.018-inch guide wire (V18, Boston Scientific, Natick, MA, USA) was advanced through one of the lumens of the catheter through the occluded segment of the superior vena cava into the inferior vena cava (Fig. 3B). The catheter was then exchanged for an 8-French vascular sheath. Venography performed via the side arm of the sheath revealed near complete occlusion of the superior vena cava at the cava-right atrial junction (Fig. 3C).

Approximately $3000 \mathrm{U}$ of heparin was administered intravenously via the sheath, and the occlusion was sequentially dilated to $14 \mathrm{~mm}$ using angioplasty balloon catheters (Fig. 3D). The underlying lesion appeared to have some degree of elasticity but ultimately, antegrade flow was reestablished into the right heart (Fig. 3E). A small amount of reflux into the azygous system persisted, indicating that complete resolution of the lesion was not achieved. Due to the location and benign etiology of the lesion, stent placement was deferred. Catheters were removed and the incision site in the chest was closed using interrupted sutures (Fig. 3F).

On subsequent follow-up, the patient experienced rapid relief of symptoms. He was maintained on anticoagulation and resolved his symptoms over the next several days.

\section{DISCUSSION}

A variety of strategies can be used to treat superior vena cava syndrome caused by indwelling catheters, including anticoagulation and thrombolysis. Thrombolysis is often the initial step and typically reduces clot burden. However, it is rarely definitive therapy-usually after thrombolysis, an underlying stenosis is revealed that requires dilation. It is generally best to eliminate the inciting process; thus, in patients with catheter-related superior

\footnotetext{
${ }^{1}$ Section of Vascular and Interventional Radiology, University of Chicago Hospitals, Chicago, Illinois.

Address for correspondence and reprint requests: Brian Funaki, M.D., Section of Vascular and Interventional Radiology, University of Chicago Hospitals, 5840 S. Maryland Avenue, MC 2026, Chicago, IL 60637.
}

Clinical Management Issues in Interventional Radiology; Guest Editor, H. Bob Smouse, M.D.

Semin Intervent Radiol 2006;23:361-365. Copyright (C) 2006 by Thieme Medical Publishers, Inc., 333 Seventh Avenue, New York, NY 10001, USA. Tel: +1(212) 584-4662.

DOI 10.1055/s-2006-957027. ISSN 0739-9529. 


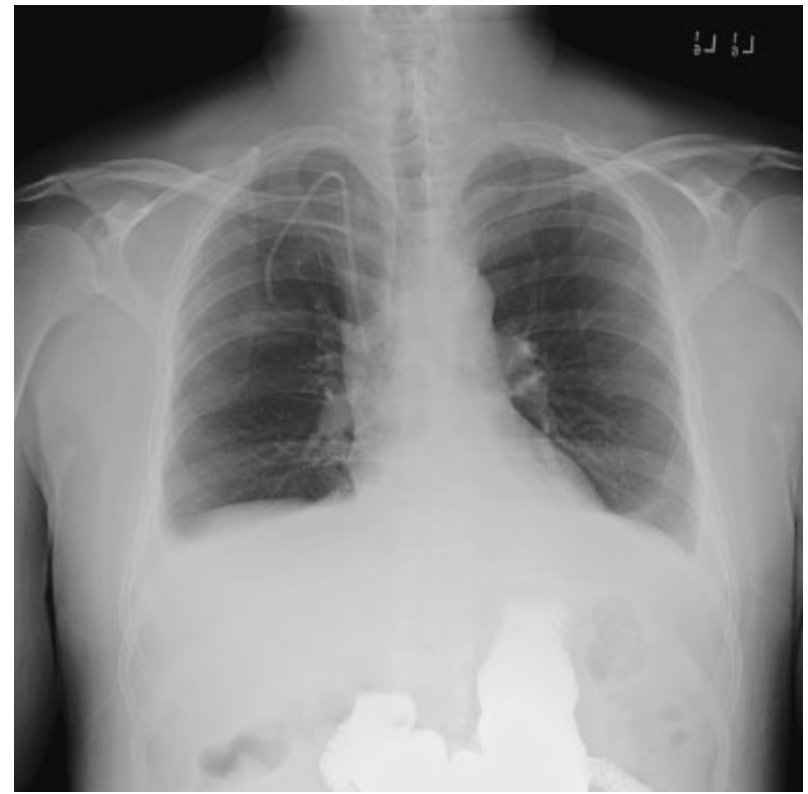

Figure 1 Percutaneous chest port insertion. Chest radiograph shows right internal jugular vein dual lumen chest port.

A
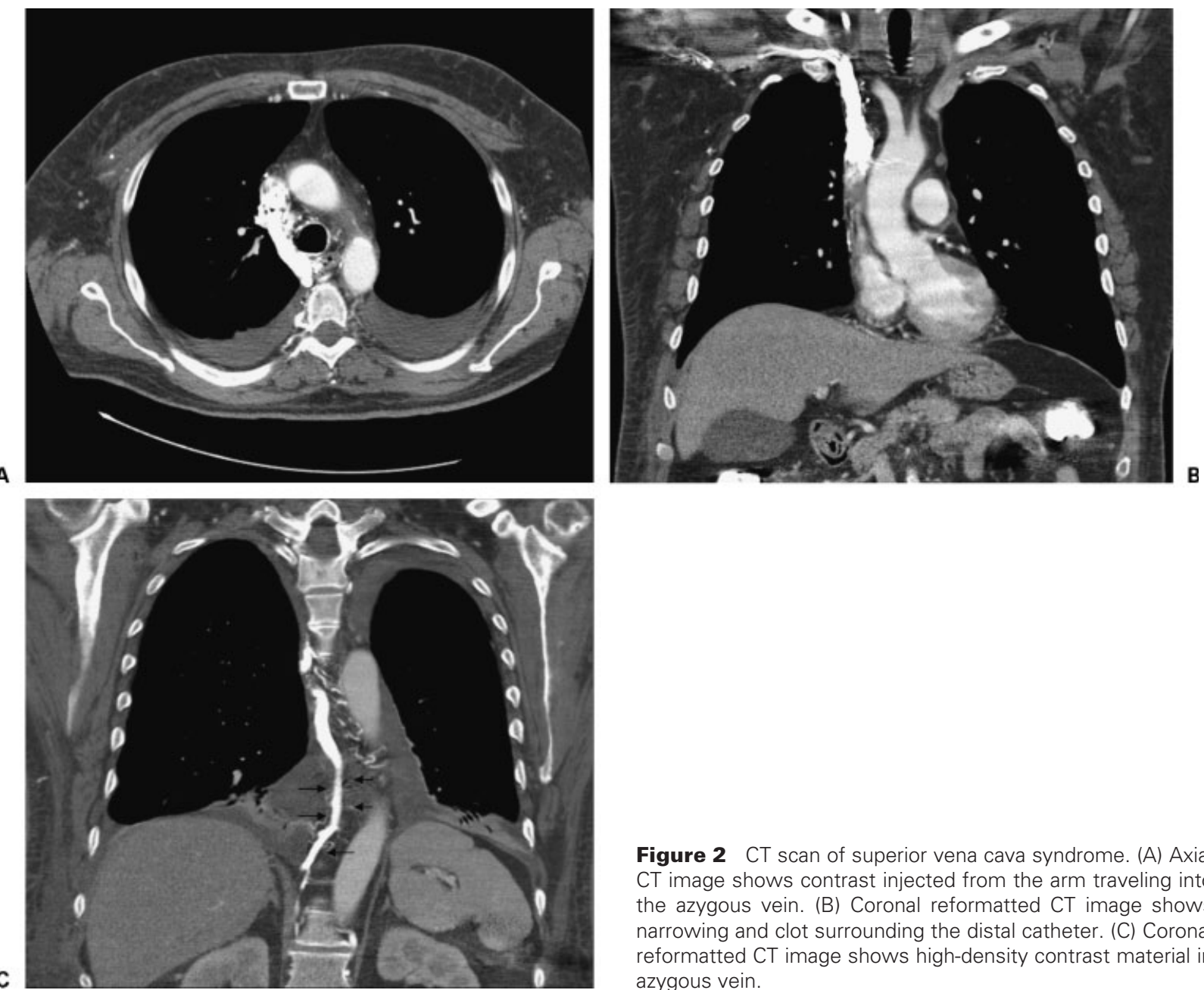

Figure 2 CT scan of superior vena cava syndrome. (A) Axia $\mathrm{CT}$ image shows contrast injected from the arm traveling into the azygous vein. (B) Coronal reformatted CT image shows narrowing and clot surrounding the distal catheter. (C) Corona reformatted CT image shows high-density contrast material in azygous vein 
A
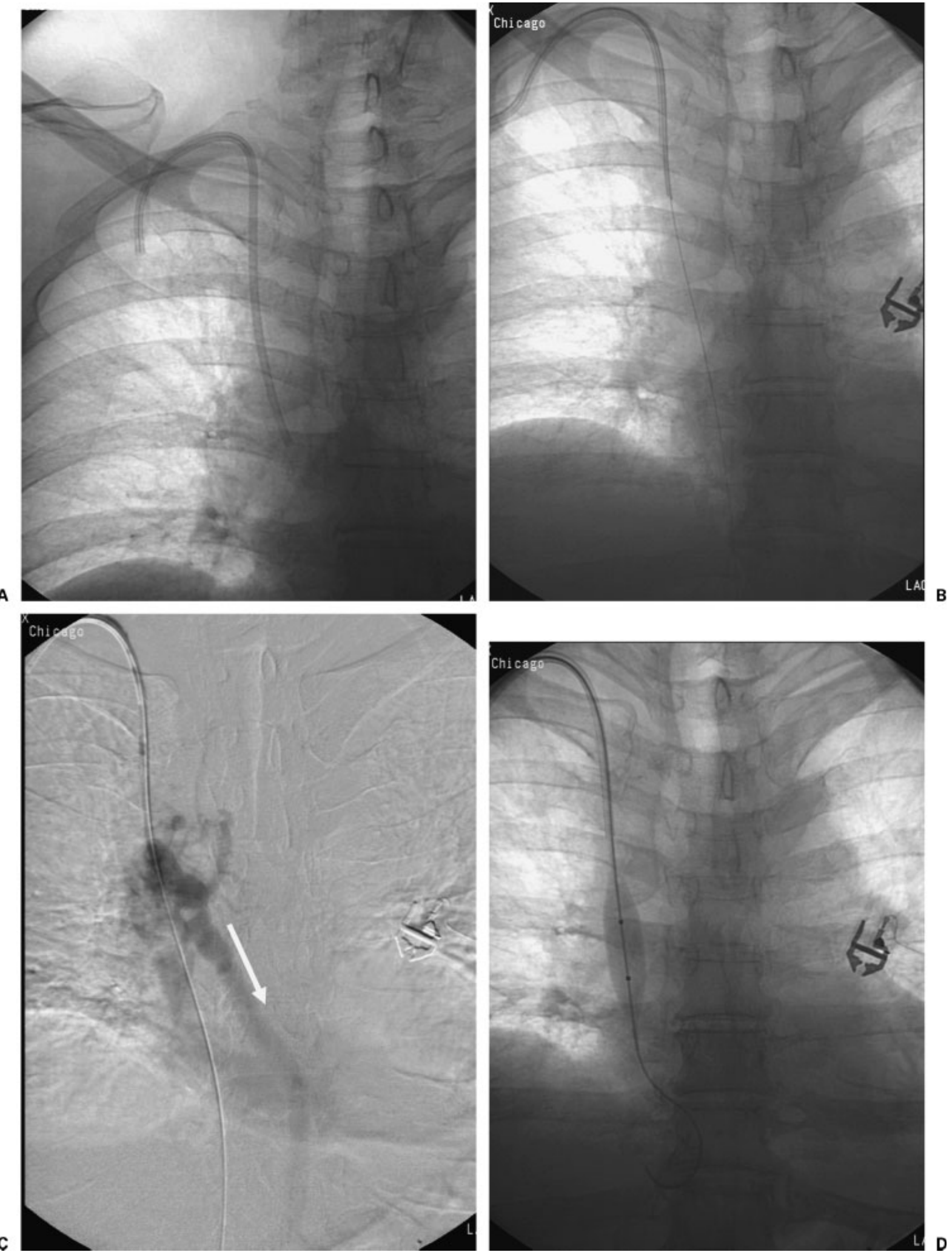

Figure 3 Superior vena cava angioplasty. (A) Initial scout view shows right dual lumen port in good position. (B) Fluoroscopic image after port dissection shows guide wire advanced through catheter into inferior vena cava. (C) Superior vena cavagram obtained via sidearm sheath shows contrast preferentially filling azygous vein (white arrow). (D) Fluoroscopic image shows 14-mm angioplasty of superior vena cava. 

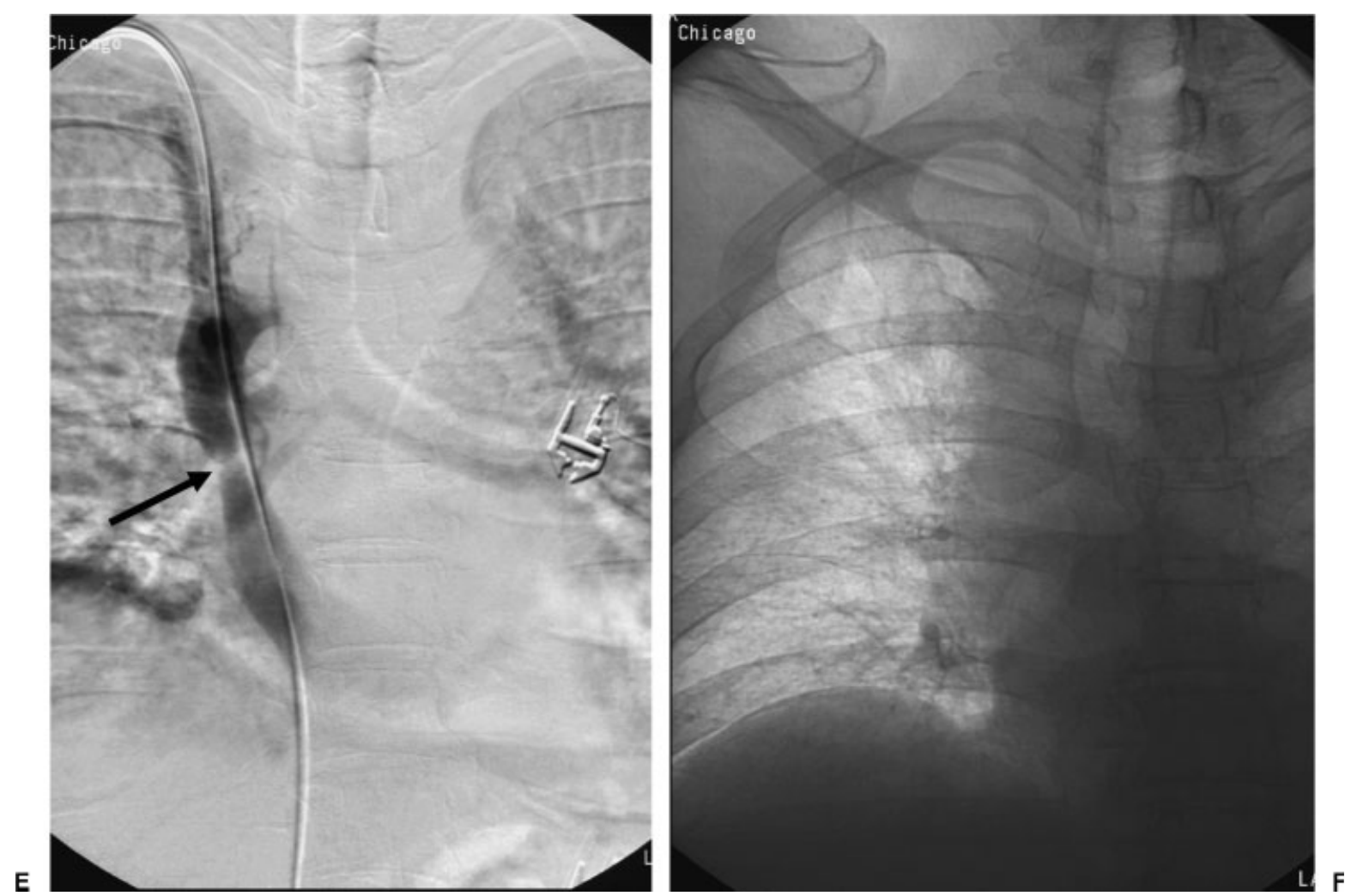

Figure 3 (con't) (E) Superior vena cavagram after angioplasty shows improved antegrade flow through superior vena cava without filling of azygous vein. Mild residual stenosis remains (black arrow). (F) Final fluoroscopic image demonstrates that the port has been removed.

vena cava syndrome, the catheter should be removed when possible. In this patient, thrombolysis did not result in improvement in symptoms, and even after port removal, angioplasty was required to restore antegrade flow in the superior vena cava. Aggressive thrombolysis was relatively contraindicated given the open incision on the patient's chest.

My approach to superior vena cava syndrome due to a nonmalignant cause differs from my approach to a malignant lesion. As a general rule, I insert stents in nearly all malignant occlusions and stenoses. In benign lesions, I will stent only when angioplasty fails to improve antegrade flow, particularly when the location of the stenosis is in close proximity to the right heart (as in this patient). One straightforward method to determine the hemodynamic result of dilation is to observe opacification of collateral veins. If after angioplasty collaterals are no longer visualized, an adequate result is assured in the vast majority of cases. On the other hand, if collaterals persist, it may be helpful to perform a pullback pressure measurement. In my experience, any gradient $>10 \mathrm{~mm}$ $\mathrm{Hg}$ requires further treatment for an optimal result.

The optimal balloon and stent diameters vary slightly from patient to patient, but typically, restoration of a diameter of 12 to $14 \mathrm{~mm}$ resolves symptoms. At minimum, $10-\mathrm{mm}$ dilation should be performed in most cases. The objective is to restore inline flow to at least one of the two jugular veins. Stents must be long enough to completely cover lesions with 1 to $2 \mathrm{~cm}$ overlap at the ends for malignant occlusions. Short self-expanding stents should be avoided because they tend to migrate ("watermelon seed") caudally or cephalad. Stents are generally chosen by personal experience and preference-no stent has proven superior to the others. I use Wallstents (Boston Scientific) most often, but for particularly fibrotic lesions, Z-stents (Cook, Bloomington, IN) provide superior radial force. Balloon expandable stents also can be used unless lesions involve both the cava and the brachiocephalic veins. In these patients, self-expanding stents that can be deployed in a curved orientation are best. One further comment regarding combined brachiocephalic vein and superior vena cava occlusion bears mentioning. It is not necessary to stent both brachiocephalic veins and superior vena cava. Symptoms will resolve as long as both the cava and one of the veins are treated adequately to establish straight-line flow to the right heart. Stenting both veins and the cava leads to a pleasing angiographic result but results in no measurable improvement in the clinical result and increases the time and cost of the procedure.

Complications are uncommon but include airway compromise, pulmonary embolism, stent malposition, and superior vena cava rupture. It is important to recall that the pericardium extends to the lower superior vena 
cava, and a tear of the lower cava can cause hemoperiocardium and tamponade. Patients with superior vena cava obstruction may be at high risk for airway occlusion when placed supine, and I routinely involve anesthesiologists for sedation and airway management.

\section{SUGGESTED READING}

Ray CE. Renal embolization. Semin Intervent Radiol 2001; 18:37-46 